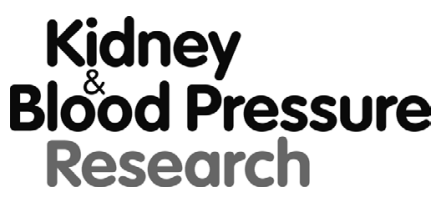

Kidney Blood Press Res 2018;43:1160-1173

DOI: $10.1159 / 000492244$

Published online: 31 July, 2018

Accepted: 20 July, 2018

This article is licensed under the Creative Commons Attribution-NonCommercial-NoDerivatives 4.0 International License (CC BY-NC-ND) (http//www.kargercom/Services/OpenAccessLicense). Usage and distribution for commercial purposes as well as any distribution of modified material requires written permission.

\title{
The Association of Fibroblast Growth Factor 23 with Arterial Stiffness and Atherosclerosis in Patients with Autosomal Dominant Polycystic Kidney Disease
}

\author{
Melahat Coban ${ }^{\mathrm{a}}$ Ayca Incia Ustun Yılmaz ${ }^{\mathrm{a}} \quad$ Emre Asilturk $^{\mathrm{b}}$ \\ antalya Training and Research Hospital, Division of Nephrology, Antalya, ${ }^{b}$ Antalya Training and \\ Research Hospital, Division of Cardiology, Antalya, Turkey
}

\section{Key Words}

Autosomal dominant polycystic kidney disease - Fibroblast growth factor-23 - Soluble klotho - Arterial stiffness - Atherosclerosis

\begin{abstract}
Background / Aims: In patients with autosomal dominant polycystic kidney disease (ADPKD), cardiovascular events are the most frequent cause of mortality and morbidity. The aim of our study is to investigate the association between serum fibroblast growth factor-23 (FGF23) and arterial stiffness (AS) as determined with brachial-ankle pulse wave velocity (baPWV) and atherosclerosis development as determined with carotid artery intima-media thickness (CA- IMT). Methods: This cross-sectional study was conducted with totally 86 ADPKD patients, $50(58.1 \%)$ female and 36 (41.9\%) male, with a mean age of $49.5 \pm 13.9$ years. Patients were compared with healthy control group with similar distribution of age and gender. AS was assessed with baPWW, and atherosclerosis development was assessed with CA-IMT. CA-IMT $>9 \mathrm{~mm}$ was considered as increased atherosclerosis. Serum FGF-23 and soluble klotho (s-KL) levels were measured with enzyme-linked immunosorbent assay. Due to skewed distribution of variables, statistical calculations of FGF-23 and s-KL were performed with log10. Results: According to the CKD stages, 46 (53.5\%) patients had stage 1-2, 32 (37.2\%) had stage 3-4, and $8(9.3 \%)$ had predialysis stage 5 disease. Mean log10FGF-23 was $2.43 \pm 0.41 \mathrm{pg} / \mathrm{mL}$, and mean log10s-KL was $1.28 \pm 0.09 \mathrm{ng} / \mathrm{mL}$. Mean baPWV was $7.48 \pm 1.68 \mathrm{~m} / \mathrm{sec}$, and mean CA-IMT was $0.63 \pm 0.14 \mathrm{~mm}$. Among patients at various stages of CKD, systolic blood pressure (SBP) ( $p$ $=0.003)$, diastolic blood pressure (DBP) $(p=0.002)$, creatinine, 1.25hydroxy $(\mathrm{OH}) 2$ VitaminD3, log10FGF-23, baPWV, CA-IMT were higher $(p<0.001)$ andlog10s-KL were lower $(p<0.001)$ in comparison to healthy individuals. FGF-23 was positively correlated with creatinine,
\end{abstract}




\section{Kidney Blood Pressure Research}

Kidney Blood Press Res 2018;43:1160-1173

\begin{tabular}{l|l}
\hline DOI: $10.1159 / 000492244$ & (C) 2018 The Author(s). Published by S. Karger AG, Basel
\end{tabular}

Published online: 31 July, 2018

www.karger.com/kb

1161

1.25(OH)2VitD3 ( $p<0.001)$, baPWV $(p=0.002)$ and CA-IMT $(p=0.005)$, and negatively correlated with eGFR $(p<0.001)$. Conclusion: In patients with ADPKD, as the disease stage advanced, serum FGF-23 levels increased while s-KL decreased. In ADPKD patients, AS and atherosclerosis development increased as compared to healthy subjects, and as CKD advanced. In ADPKD patients, the effect of serum FGF-23 on the development of AS and atherosclerosis in peripheral vessels is independent of $\mathrm{s}-\mathrm{KL}$.

(C) 2018 The Author(s)

Published by S. Karger AG, Basel

\section{Introduction}

Autosomal dominant polycystic kidney disease (ADPKD) is the most common inherited renal disease. ADPKD is responsible for $5-10 \%$ of cases with end stage renal disease (ESRD) [1]. The expansion of the fluid-filled cysts over time causes the kidneys to expand dramatically in size. Around the fourth decade, the estimated glomerular filtration rate (eGFR) starts to decrease by $4.4-5.9 \mathrm{~mL} / \mathrm{min}$ per year and ESRD development is seen at 6th decade.

Fibroblast growth factor-23 (FGF-23) is a hormone mainly synthesized from osteocytes and to a lesser degree form osteoblasts, endocrine organs, heart, hypothalamus, and thalamus. Soluble klotho (s-KL) functions as FGF-23 co-receptor [2]. FGF-23 binds to FGF receptors in the kidneys in the presence of s-KL, inhibits sodium (Na)-dependent phosphate $(\mathrm{P})$ carriers $\mathrm{NaP}-$ IIa and NaP-IIc in the proximal tubule leading to renal $\mathrm{P}$ excretion. It also decreases 1.25hydroxy $(\mathrm{OH}) 2$ Vitamin(Vit)D3 synthesis by inhibiting the enzyme 1- $\alpha$ hydroxylase.

In patients with chronic kidney disease (CKD), serum FGF-23 levels increase starting from the early stages. Liu et al. reported that elevated serum P, intact parathyroid hormone (iPTH) levels and decreased serum 1.25hydroxy $(\mathrm{OH}) 3$ Vitamin(Vit)D3 levels were accompanied by an increase in serum FGF-23 levels in CKD patients [3]. Gutierrez et al. [4] reported that elevated serum P levels were associated with elevated serum FGF-23 levels in patients with early stage CKD. John et al. [5] reported that as the P excretion load per nephron increased in CKD patients, serum FGF-23 levels increased to overcome this burden. Yildiz et al. [6] reported that elevated serum FGF-23 levels were found in early stage ADPKD patients with normal creatinine levels in comparison to healthy individuals.

In patients with CKD, arterial stiffness (AS) is one the most common vascular pathologies. Tsuchikura et al. reported that the determination of AS development was convenient and reproducible with brachial-ankle pulse wave velocity (baPWV) device, and that it showed good correlation with aorticpulse wave velocity, which is regarded as the gold standard. Covic et al. [7] proposed that utilization of pulse wave velocity determined by baPWV device could be valuable in determination of AS development in CKD patients. London et al. [8] reported increased AS development in CKD patients in comparison to healthy individuals.

In patients with ADPKD, cardiovascular (CV) complications are frequent causes of increased mortality. In ADPKD patients, increased CV events start to develop from the early stages of the disease. Kocyigit et al. [9] reported increased AS development in ADPKD patients compared to healthy individuals. Borresen et al. [10] reported increased AS development independent of CKD development in patients with ADPKD.

In literature, there is a limited number of studies investigating increased FGF-23 levels and AS development. In the general population, the association between increased serum FGF-23 levels and AS development is known to be particularly more profound in those with impaired renal function tests. Scialla et al. reported that there was a relationship between increased serum FGF- 23 and AS development in CKD patients [11]. On the contrary, Manghat et al. reported that there was no relationship between serum FGF-23 levels and AS development in predialysis CKD patients [12]. Similarly, Ford et al. reported that there was no relationship between serum FGF-23 levels and AS development in early stage CKD patients [13].

Atherosclerosis is a systemic disease affecting blood vessels of all sizes, and medium sized elastic arteries in particular. Measurement of carotid artery intima-media thickness 


\section{Kidney Blood Pressure Research}

Kidney Blood Press Res 2018;43:1160-1173

\begin{tabular}{l|l}
\hline DOI: $10.1159 / 000492244$ & (C) 2018 The Author(s). Published by S. Karger AG, Base
\end{tabular}

Published online: 31 July, 2018

www.karger.com/kb

(CA-IMT) via ultrasonography is regarded as a non-invasive and convenient method for assessment of atherosclerosis. Poredos et al. [14] proposed that increased CA-IMT value is an indicator of atherosclerosis and is correlated with peripheral arterial diseases. Kocaman et al. [15] reported increased atherosclerosis development in early stage ADPKD patients compared to healthy individuals. Sag et al. [16] reported that atherosclerosis development as determined by CA-IMT was increased in ADPKD patients compared to healthy individuals. Turkmen et al. reported increased atherosclerosis development in ADPKD patients starting from the early stages of CKD [17].

Studies investigating the relationship between FGF-23 and atherosclerosishave controversial results. In their mouse study, Larrson et al. [18] reported that there was no relationship between increased serum FGF-23 and atherosclerosis development. Scialla et al. [19] reported that there was no relationship between increased serum FGF-23 and increased atherosclerosis development in CKD patients. On the contrary, Nakayawa et al. [20] reported that there was an association between increased serum FGF-23 levels and atherosclerosis development in CKD patients.

It is known that in CKD patients as the disease advances, serum FGF-23 levels increase while serum s-KL levels decrease. In CKD patients, increased serum FGF-23 levels are thought to be associated with increased CV diseases. There is a limited number of studies that investigated serum FGF-23 and s-KL levels in patients with ADPKD, which is a genetic disease characterized by presence of multiple cysts in kidneys, and the association of these with increased AS and atherosclerosis development, and these studies have contradicting results. Furthermore, these studies were conducted with ADPKD patients at early stage and with normal creatinine levels. For that reason, the aim of our study was to investigate the association of serum FGF-23 levels with AS as determined by baPWV, and atherosclerosis development as determined by CA-IMT in adult ADPKD patients at various predialysis CKD stages.

\section{Materials and Methods}

\section{Patient selection}

This cross-sectional study was conducted inNephrology outpatient clinic of Antalya Training and Research Hospital between November 2016 and September 2017, with a total of 86 patients diagnosed with autosomal dominant policystic kidney disease (ADPKD), of which 50 (58.1\%) were female (mean age $=49.5 \pm 13.9$ years) and $36(41.9 \%)$ were male (mean age $=47.8 \pm 13.9$ years). Patients were compared with 47healthy controls with similar distribution of age and gender, who did not have any chronic disease or history of medication use. The study included patients diagnosed with ADPKD based on family history, clinical findings andultrasonography, who were older than 18 years of age, and who did not have history of known cardiac disease, peripheral vessel disease, cardiac intervention, or renal replacement therapy. Study exclusion criteria were less than 1 year life expectancy, active infection or malignancy, previous history of cardiac intervention (coronary bypass, stent, cardiac pacemaker) or cardiac disease (left ventricular (LV) ejection fraction assessed with echocardiography $<45 \%$, heart valvular disease, cardiomyopathy), peripheral vessel disease, history of renal transplantation or dialysis, and patient's refusal to participate in the study. All participants were informed about the purpose of the study, and written consent was obtained from volunteers. The study was approved by Antalya Training and Research Hospital Ethics Committee.

\section{Data collection and laboratory measurements}

A detailed medical history was obtained and physical examination was performed in the entire study group. Demographic characteristics (gender, age, body mass index), comorbid diseases and antihypertensive medications used were recorded. Venous blood samples taken after 8 hours of fasting were centrifuged and stored at $-80^{\circ} \mathrm{C}$. Serum creatinine, calcium (Ca), phosphate (P) and high sensitive C reactive protein (hs-CRP) levels were determined spectrophotometrically using Beckman coulter AU5800 (Beckman coulter Instrumentation, San Diego, CA, USA) analyzer. Intact parathyroid hormone (iPTH) level was measured with 


\section{Kidney Blood Pressure Research}

Kidney Blood Press Res 2018;43:1160-1173

\begin{tabular}{l|l}
\hline DOI: $10.1159 / 000492244$ & (C) 2018 The Author(s). Published by S. Karger AG, Basel
\end{tabular}

Published online: 31 July, 2018

www.karger.com/kbr

Kidney Disease

chemiluminescence method on Beckman coulter DxI800 (Beckman coulter Instrumentation, San Diego, CA, USA) analyser. Fibroblast growth factor-23 (FGF-23), soluble-Klotho (s-KL) (Elabscience, Shanghai, China) and 1.25hydroxy(OH)2Vitamin(Vit)D3 (BioassayTechnology Laboratory, Shangai, China) levels were measured with enzyme-linked immunosorbent assay (ELISA) kits. 25(OH)VitD3 level was measured with chemiluminescence assay using Liason (DiaSorin, MN,USA) analyser. For all parameters, the inter- and intraassay coefficients of variations were $<10 \%$; measurement ranges and analytical sensitivities were 15.6$1000 \mathrm{pg} / \mathrm{mL}$ and $9.38 \mathrm{pg} / \mathrm{mL}$ for FGF-23, 0.31-20 ng/mL and 0.19 ng/mL for s-KL, and 0.2-60 ng/mL and $0.07 \mathrm{ng} / \mathrm{mL}$ for $1.25(\mathrm{OH}) 2 \mathrm{VitD} 3$, respectively.

Spot urinary protein-creatinine ratio (UPCR) was calculated from the second morning urine sample collected after 8 hours of fasting. Kidney functions were assessed with serum cre, estimated glomerular filtration rate (eGFR) and UPCR measurement. Patients' eGFR values were calculated according to the criteria defined by Levey et al. [21]. Patients were divided into 3 chronic kidney disease (CKD) stages according to their eGFR values: Stage 1-2 CKD: eGFR

$=60-110 \mathrm{~mL} / \mathrm{min} / 1.73 \mathrm{~m} 2$; Stage $3-4$ CKD: eGFR $=15-59 \mathrm{~mL} / \mathrm{min} / 1.73 \mathrm{~m} 2$, and predialysis

stage 5 CKD: eGFR $<15 \mathrm{~mL} / \mathrm{min} / 1.73 \mathrm{~m} 2$. Blood pressure measurements were performed by the same nurse after 15 minutes of rest. Hypertension (HT) was defined as previous history of HT, or antihypertensive medication use, or systolic blood pressure (SBP)/diastolic blood pressure (DBP) $\geq 140 / 90 \mathrm{mmHg}$.

\section{Measurement of Arterial Stiffness}

Arterial stiffness (AS) development with brachial-ankle pulse wave velocity (baPWV) was determined in the entire patient and control groups after 15 minutes resting in supine position, using brachial-ankle index (ABI)-form instrument (D-52222, 2007, Stolberg, Germany). As described in the previous study [22] the blood pressure measurements at both arms and legs were calculated automatically and at the same time. The PWV value was calculated by determining the delay between localized blood pressure curves at two different points of the arterial system. The interval between the start and end of the brachial and ankle waves obtained from brachial and tibial arteries were determined as transition time (TT). The transition distance between brachial and ankle was determined according to weight. The distance between the suprasternal notch-brachium (LB) was calculated with the formula $0.2195 \times$ height of the patient (in $\mathrm{cm}$ ) -2.0734 , whereas the distance between suprasternal notch-ankle (LA) was calculated by the formula $0.2195 \mathrm{x}$ height of the patient $(\mathrm{in} \mathrm{cm})+12.328$. The baPWV value was determined by the formula= (LA-LB)/TT.

\section{Measurement of Atherosclerosis}

Carotid artery intima-media thickness (CA-IMT) measurements were performed by the same radiologist, using a high-resolution, 2-5 $\mathrm{mHz}$ linear array probe with Hitachi Hi-Vision avius (Tokyo, Japan) ultrasonography (USG) instrument. Measurements were made when all participants were lying in the supine position and the neck was in the slightly extended state and the head turned to the other side. Measurements were made at 3 different points: the right and left common carotid arteries (CCA), bifurcation point and the first $2 \mathrm{~cm}$ segment of the internal carotid artery. The CA-IMT value was determined at exactly longitudinal plane, where anterior and posterior walls were visualized together, measuring the distance between the hyperechogenic reflection at the lumen of intima at the posterior wall, and the hyperechogenic reflection formed by the media-adventitia at deep media layer. CA-IMT $>9 \mathrm{~mm}$ was considered as atherosclerosis development.

\section{Statistical Analysis}

Continuous variables were expressed as mean \pm standard deviation, while categorical variables were expressed as frequency and percentage (\%). In comparison of patient characteristics of ADPKD patients at different CKD stages with healthy control group, Kruskal-Wallis and Bonferroni-Dunn Post-hoc test was performed for non-normally distributed data. In comparison of patient characteristics according to mean serum FGF-23 and s-KL levels, Mann-Whitney U test was used for quantitative variables with non-normal distribution. In correlation analysis of patient characteristics with FGF-23; Spearman rank test was used for non-normal distributed data and Pearson test was used for normal distributed data. $\mathrm{P}<0.05$ was considered statistically significant. 


\section{Kidney \\ Blood Pressure Research}

Kidney Blood Press Res 2018;43:1160-1173

\begin{tabular}{l|l}
\hline DOI: $10.1159 / 000492244$ & (C) 2018 The Author(s). Published by S. Karger AG, Basel
\end{tabular}

Published online: 31 July, 2018

www.karger.com/kbr

1164

Coban et al.: Fibroblast Growth Factor 23 Levels in Autosomal Dominant Polycystic

Kidney Disease

\section{Results}

The study included totally 86 ADPKD patients, 50 (58.1\%) women with a mean age of $49.5 \pm 13.9$ years, and $36(41.9 \%)$ men with a mean age of $47.8 \pm 13.9$ years. Mean SBP was $129.2 \pm 173 \mathrm{mmHg}$, and mean DBP was $86.5 \pm 10.7 \mathrm{mmHg} .6(7 \%)$ patients had DM and 56 $(65.1 \%)$ patients had HT. Anti-hypertensive medications used by patients were angiotensin converting enzyme inhibitor in $15(17.4 \%)$ patients, angiotensin II receptor blockerin 27 $(31.4 \%)$ patients, Calciumchannel blocker in $20(23.3 \%)$ patients, alpha blocker in $4(4.7 \%)$ patients, beta-blocker in $15(17.4 \%)$ patients, diuretic in $4(4.7 \%)$ patients, and other antihypertensive medications in $3(3.5 \%)$ patients. According to the eGFR values, patients were divided into 3 different CKD categories: 46 (53.5\%) patients had stage 1-2 CKD, 32 (37.2\%) patients had stage $3-4$, and $8(9.3 \%)$ patients had predialysis stage 5 CKD. $19(22.1 \%)$ patients were using Vit D/Vit D analogue, and 4 (4.7\%) patients were using oral phosphate binders. Mean creatinine was $1.52 \pm 0.87 \mathrm{mg} / \mathrm{dL}$, and mean eGFR was $61 \pm 30 \mathrm{~mL} / \mathrm{min} / 1.73$ m2. Mean hs-CRP, iPTH, CasXPs product, 25(OH)VitD3 and 1.25(OH)2VitD3 levels were $2.72 \pm 2.55 \mathrm{mg} / \mathrm{L}, 82 \pm 50 \mathrm{pg} / \mathrm{mL}, 29.24 \pm 6.16 \mathrm{mg} / \mathrm{dL}, 19.75 \pm 13.49 \mathrm{ng} / \mathrm{mL}$, and 27.57 $\pm 20.82 \mathrm{ng} / \mathrm{mL}$, respectively. Mean serum $\log 10 \mathrm{FGF}-23$ was $2.43 \pm 0.41 \mathrm{pg} / \mathrm{mL}$, and mean serum log10s-KL was $1.28 \pm 0.09 \mathrm{ng} / \mathrm{mL}$. Mean baPWV was $7.48 \pm 1.68 \mathrm{~m} / \mathrm{sec}$, and mean CAIMT was $0.63 \pm 0.14 \mathrm{~mm} .6(7 \%)$ patients had CA-IMT $>9 \mathrm{~mm}$.Patients were compared to 47 healthy controls $(22(46.8 \%)$ male/25 (53.2\%) female) with a mean age of $45.7 \pm 7.2$ years. Mean SBP was $118.6 \pm 11.7 \mathrm{mmHg}$, and mean DBP was $78.4 \pm 9.7 \mathrm{mmHg}$. Mean creatinine was $0.8 \pm 0.1 \mathrm{mg} / \mathrm{dL}$, and mean eGFR was $91.4 \pm 6.9 \mathrm{~mL} / \mathrm{min} / 1.73 \mathrm{~m}^{2}$. Mean hs-CRP, iPTH, CasXPs product, 25(OH)VitD3 and 1.25(OH)2VitD3 levels were $2.6 \pm 2.8 \mathrm{mg} / \mathrm{L}, 53 \pm 25 \mathrm{pg} /$ $\mathrm{mL}, 31.4 \pm 4.8 \mathrm{mg} / \mathrm{dL}, 20.8 \pm 10.9 \mathrm{ng} / \mathrm{mL}$, and $19.5 \pm 15.4 \mathrm{ng} / \mathrm{mL}$, respectively. Mean serum $\log 10 \mathrm{FGF}-23$ was $2.08 \pm 0.3 \mathrm{pg} / \mathrm{mL}$, and mean serum $\log 10 \mathrm{~s}-\mathrm{KL}$ was $1.1 \pm 1 \mathrm{ng} / \mathrm{mL}$. Mean baPWV was $6.6 \pm 0.9 \mathrm{~m} / \mathrm{sec}$, and mean CA-IMT was $0.65 \pm 0.66 \mathrm{~mm}$ (Table 1 ).

In comparison to healthy controls, patients at various predialysis CKD stages had significantly higher SBP ( $p=0.003)$, DBP $(p=0.002)$, hs-CRP $(p=0.001)$, creatinine, iPTH, 1.25(OH)2VitD3, log10FGF-23, baPWV,and CA-IMT ( $<<0.001)$, and significantly lower eGFR and $\log 10 \mathrm{~s}-\mathrm{KL}(\mathrm{p}<0.001)$. There was no significant difference between the two groups regarding CasXPs product, or 25(OH)VitD3 values ( $\mathrm{p}>0.05)$. ADPKD patients with stage 1-2 had higher eGFR, 1.25(OH)2VitD3, log10s-KL, and lower creatinine, hs-CRP, iPTH, log10FGF-23, baPWV and CA-IMT than patients with predialysis stage 5 CKD (Table 2).

Mean FGF-23 level was 285.03 pg/mL. Patients with FGF-23 $>285.03$ had significantly higher creatinine, iPTH $(\mathrm{p}=0.001)$, baPWV $(\mathrm{p}=0.004)$ and CA-IMT $(\mathrm{p}=0.003)$, and lower eGFR $(\mathrm{p}=0.001)$ and $1.25(\mathrm{OH}) 2$ VitD3 $(\mathrm{p}<0.001)$ compared to patients with FGF-23 $\leq$ 285.03. There was no significant difference between the two groups regarding hs-CRP, CasXPs product, or s- KL (all p > 0.05) (Table 3).

Serum $\log 10 \mathrm{FGF}-23$ levels showed positive correlation with iPTH (Fig. 1), 1.25(OH)2VitD3 (Fig. 2) (all p < 0.001), baPWV (p =0.002) (Fig. 3) and CA-IMT ( $p=0.005$ ) (Fig. 4), and negative correlation with eGFR ( $p<0.001)$ (Fig. 5). There was no correlation between log10FGF-23 and hs-CRP (Fig. 6), CasXPs product (Fig. 7) or 25(OH)VitD3 (Fig. 8) (all $p>0.05$ ) in patients. Serum log10FGF-23 levels showed positive correlation with creatinine $(p=0.043)$ and negative correlation with eGFR $(p=0.022)$ in healthy controls. There was no correlation between log10FGF-23 and iPTH, 25(OH)VitD3, 1.25(OH)2VitD3, baPWV or CA- IMT (all p > 0.05) in healthy controls (Table 4).

Mean s-KL value was $19.26 \mathrm{ng} / \mathrm{mL}$. Patients with s-KL $>19.26$ did not differ from patients with s-KL $\leq 19.26$ regarding baPWV (Fig. 9) and CA-IMT (Fig. 10) (all p > 0.05) (Table 5). 


\section{Kidney Blood Pressure Research}

Coban et al:: Fibroblast Growth Factor 23 Levels in Autosomal Dominant Polycystic

Table 1. Clinical, demographic characteristics, laboratory and baPWV values of patients and healthy control group. Data are presented as n (\%), mean \pm S.D. Abbreviations: BMI, body mass index; SBP, systolic blood pressure; DBP, diastolic blood pressure; DM; diabetes mellitus; HT, hypertension; ACE inh, angiotensin converting enzyme inhibitor; ARB, angiotensin II receptor blocker; $(\mathrm{OH})$ Vit, (hidroksi)vitamin; eGFR, estimated glomerular filtration rate; hs-CRP, high sensitive $\mathrm{C}$ reactive protein; $\mathrm{PPTH}$, intact parathyroid hormone; Ca, serum calcium; Ps, serum phosphate; FGF-23, fibroblast growth factor-23; s-KL, soluble klotho; baPWV, brachial-ankle pulse wave velocity; CA-IMT, carotid artery intima-media thickness; S.D., standard deviation

\begin{tabular}{|c|c|c|}
\hline Parameter & $\begin{array}{c}\text { Patients }(\mathrm{n}=86) \\
\text { Mean } \pm \text { S.D. } / \mathrm{n}(\%)\end{array}$ & $\begin{array}{l}\text { Healthy control group }(n=47) \\
\text { Mean } \pm \text { S.D. } / n(\%) .\end{array}$ \\
\hline Age (years) Male/Female & $\begin{array}{c}49 \pm 14 \\
47.8 \pm 13.9 / 49.5 \pm 13.9\end{array}$ & $\begin{array}{c}45.7 \pm 7.2 \\
47.3 \pm 6.1 / 44.3 \pm 7.9\end{array}$ \\
\hline Male/Female & $36(41.9 \%) / 50(58.1 \%)$ & $22(46.8 \%) / 25(53.2 \%)$ \\
\hline BMİ $\left(\mathrm{kg} / \mathrm{m}^{2}\right)$ & $26.8 \pm 4.9$ & $27.1 \pm 4.6$ \\
\hline $\mathrm{SBP}(\mathrm{mmHg}) / \mathrm{DBP}(\mathrm{mmHg})$ & $129.2 \pm 173 / 86.5 \pm 10.7$ & $118.6 \pm 11.7 / 78.4 \pm 9.7$ \\
\hline DM HT & $\begin{array}{c}6(7 \%) \\
56(65.1 \%)\end{array}$ & \\
\hline \multicolumn{3}{|l|}{ Use of antihypertensive drugs } \\
\hline $\begin{array}{l}\text { ACE inh/ARB } \\
\text { Calcium channel blocker } \\
\text { Alpha-blocker/Beta-blocker } \\
\text { Diuretic } \\
\text { Others } \\
\text { Stage } 1-2 \\
\text { Stage } 3-4 \\
\text { Predialysis stage } 5 \\
\text { Use of Vit D/Vit D analog } \\
\text { Use of oral phosphate binder }\end{array}$ & $\begin{array}{c}15(17.4 \%) / 27(31.4 \%) \\
20(23.3 \%) \\
4(4.7 \%) / 15(17.4 \%) \\
4(4.7 \%) \\
3(3.5 \%) \\
46(53.5 \%) \\
32(37.2 \%) \\
8(9.3 \%) \\
19(22.1 \%) \\
4(4.7 \%)\end{array}$ & \\
\hline Creatinine (mg/dL) & $1.52 \pm 0.87$ & $0.8 \pm 0.1$ \\
\hline eGFR $\left(\mathrm{mL} / \mathrm{min} / 1.73 \mathrm{~m}^{2}\right)$ & $61 \pm 30$ & $91.4 \pm 6.9$ \\
\hline hs-CRP (mg/L) & $2.72 \pm 2.55$ & $2.6 \pm 2.8$ \\
\hline iPTH (pg/mL) & $82 \pm 50$ & $53 \pm 25$ \\
\hline CasXPs product (mg/dL) & $29.24 \pm 6.16$ & $31.4 \pm 4.8$ \\
\hline 25(OH)VitD3 (ng/mL) & $19.75 \pm 13.49$ & $20.8 \pm 10.9$ \\
\hline $1.25(\mathrm{OH}) 2 \mathrm{VitD} 3(\mathrm{ng} / \mathrm{mL})$ & $27.57 \pm 20.82$ & $19.5 \pm 15.4$ \\
\hline Log10FGF-23 (pg/mL) & $2.43 \pm 0.41$ & $2.08 \pm 0.3$ \\
\hline Log10s-KL (ng/mL) & $1.28 \pm 0.09$ & $1.1 \pm 1$ \\
\hline baPWV (m/sec) & $7.48 \pm 1.68$ & $6.6 \pm 0.9$ \\
\hline CA-IMT $(\mathrm{mm})>0.9$ & $\begin{array}{l}0.63 \pm 0.14 \\
\quad 6(7 \%)\end{array}$ & $0.65 \pm 0.66$ \\
\hline
\end{tabular}

Table 2. Comparison of patients at various predialysis stages of CKD with the healthy control group with regard to patient characteristics. Data are presented as median (min-max). Kruskal-Wallis with BonferroniDunn post-hoc test

\begin{tabular}{|c|c|c|c|c|c|}
\hline Parameter & Stage $1-2(n=46)$ & Stage $3-4(n=32)$ & Predialysisst age $5(n=8)$ & Healthy control group $(n=47)$ & p-value \\
\hline SBP (mmHg) & $126(95-159)$ & $128(100-192)$ & $128(100-192)$ & $118(90-141)$ & 0.003 \\
\hline $\mathrm{DBP}(\mathrm{mmHg})$ & $84(71-107)$ & $87(62-108)$ & $85(64-108)$ & $77(48-99)$ & 0.002 \\
\hline Creatinine $(\mathrm{mg} / \mathrm{dL})$ & $0.9(0.6-1.32)$ & $1.89(1.25-4.19)$ & $2.63(1.8-4.1)$ & $0.8(0.66-1.02)$ & $<0.001$ \\
\hline $\mathrm{eGFR}\left(\mathrm{mL} / \mathrm{min} / 1.73 \mathrm{~m}^{2}\right)$ & $86(61-122)$ & $37(16-58)$ & $22(17-30)$ & $91(71-110)$ & $<0.001$ \\
\hline hs-CRP (mg/L) & $1.15(0.16-10.2)$ & $3.03(0.16-10.2)$ & $3.86(1.47-10.2)$ & $1.36(0.16-10.2)$ & 0.001 \\
\hline iPTH $(\mathrm{pg} / \mathrm{mL})$ & $48(16-135)$ & $91(27-219)$ & $156(70-219)$ & $51(14-158)$ & $<0.001$ \\
\hline CasXPs product (mg/dL) & $29.76(18.27-61)$ & $28.5(17.1-38)$ & $28.35(20.46-38)$ & $30.72(23-44.16)$ & 0.094 \\
\hline 25(OH)VitD3 (ng/mL) & $15.6(4-88.7)$ & $18.9(4.3-62.8)$ & $18.85(4.3-32.5)$ & $18.1(6.93-58.9)$ & 0.360 \\
\hline $1.25(\mathrm{OH}) 2 \mathrm{VitD} 3(\mathrm{ng} / \mathrm{mL})$ & $41.25(14.51-78.86$ & $23.56(8.22-87.7)$ & $15.67(3.04-77.44$ & $12.83(2.08-56.54)$ & $<0.001$ \\
\hline $\log 10 \mathrm{FGF}-23(\mathrm{pg} / \mathrm{mL})$ & $2.31(1.36-3.02)$ & $2.52(1.45-3.14)$ & $2.71(2.34-3.12)$ & $2.05(1.58-2.79)$ & $<0.001$ \\
\hline $\log 10 \mathrm{~s}-\mathrm{KL}(\mathrm{ng} / \mathrm{mL})$ & $1.27(1.05-1.43)$ & $1.24(1.05-1.43)$ & $1.14(0.9-1.33)$ & $1.3(1.19-1.42)$ & $<0.001$ \\
\hline baPWV (m/sec) & $6.55(4.8-10.5)$ & $8.05(5.5-11.6)$ & $8.75(6.8-11.6)$ & $6.6(4.8-9)$ & $<0.001$ \\
\hline CA-IMT (mm) & $0.55(0.5-1.1)$ & $0.7(0.5-1.2)$ & $0.7(0.5-1.1)$ & $0.5(0.5-5)$ & $<0.001$ \\
\hline
\end{tabular}




\section{Kidney Blood Pressure Research}

Coban et al.: Fibroblast Growth Factor 23 Levels in Autosomal Dominant Polycystic Kidney Disease

Table 3. Comparison of patients characteristics according to mean FGF-23 values. Data are presented as median(min-max). Mann-Whitney U test

\begin{tabular}{lccc}
\hline Parameter & $\begin{array}{c}\text { FGF-23 } 285.03 \mathrm{pg} / \mathrm{mL} \\
(\mathrm{n}=44)\end{array}$ & $\begin{array}{c}\text { FGF-23 }>285.03 \mathrm{pg} / \mathrm{mL} \\
(\mathrm{n}=42)\end{array}$ & $\mathrm{p}$-value \\
\hline Creatinine $(\mathrm{mg} / \mathrm{dL})$ & $1(0.6-2.29)$ & $1.53(0.69-4.19)$ & 0.001 \\
eGFR $\left(\mathrm{mL} / \mathrm{min} / 1.73 \mathrm{~m}^{2}\right)$ & $74(22-120)$ & $42(16-122)$ & 0.001 \\
hs-CRP $(\mathrm{mg} / \mathrm{L})$ & $1.72(0.16-9.08)$ & $1.97(0.16-10.2)$ & 0.876 \\
iPTH $(\mathrm{pg} / \mathrm{mL})$ & $52(18-185)$ & $88(16-219)$ & 0.001 \\
CasXPs product $(\mathrm{mg} / \mathrm{dL})$ & $28.82(18.27-37.84)$ & $29.42(17.1-61)$ & 0.904 \\
25(OH)VitD3 (ng/mL) & $17(4-62.8)$ & $18.6(4.3-88.7)$ & 0.677 \\
1.25(OH)2VitD3 (ng/mL) & $25.61(8.12-87.7)$ & $14.91(3.04-58.75)$ & $<0.001$ \\
baPWV $(\mathrm{m} / \mathrm{sec})$ & $6.8(5-11.6)$ & $7.8(4,8-10.5)$ & 0.004 \\
CA-IMT $(\mathrm{mm})$ & $0.6(0.5-1.2)$ & $0.7(0.5-1.1)$ & 0.003 \\
\hline
\end{tabular}

Fig. 1. Correlation between FGF-23 and $\mathrm{PPTH}(\mathrm{R}=0.445, \mathrm{p}<0.001)$.

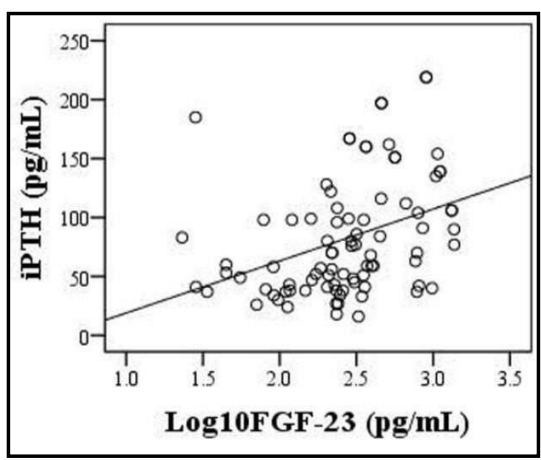

Fig. 2. Correlation between FGF-23 and $1.25(\mathrm{OH}) 2 \mathrm{VitD} 3(\mathrm{R}=$ $0.481, \mathrm{p}<0.001$ ).

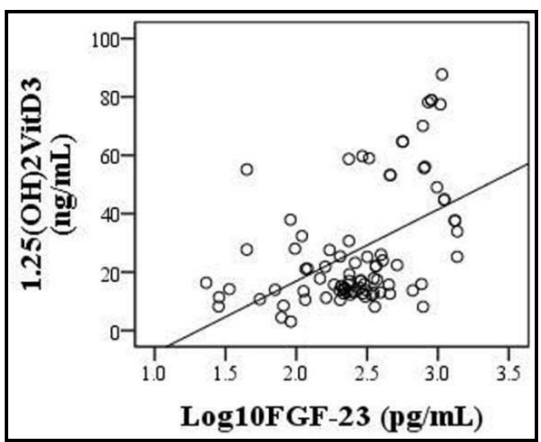

Fig. 3. Correlation between FGF- 23 and baPWV in patients $(\mathrm{R}=$ $0.337, \mathrm{p}=0.002$ ).

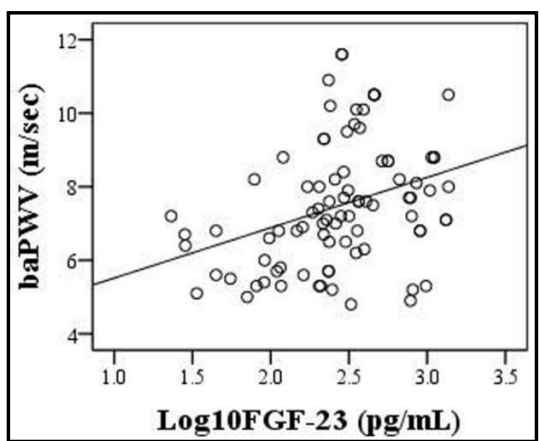




\section{Kidney Blood Pressure Research}

Fig. 4. Correlation between FGF-23 and CA-IMT in patients $(\mathrm{R}=$ $0.298, \mathrm{p}=0.005)$.

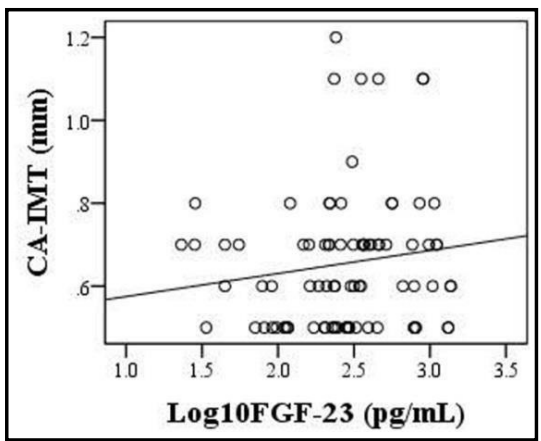

Fig. 5. Correlation between FGF-23 and eGFR $(\mathrm{R}=-0.438$, $\mathrm{p}<0.001$ ).

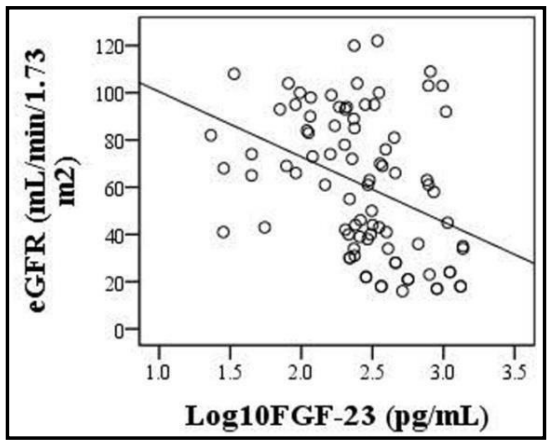

Fig. 6. Correlation between FGF-23 and hs-CRP $(\mathrm{R}=0.076, \mathrm{p}=$ 0.489).

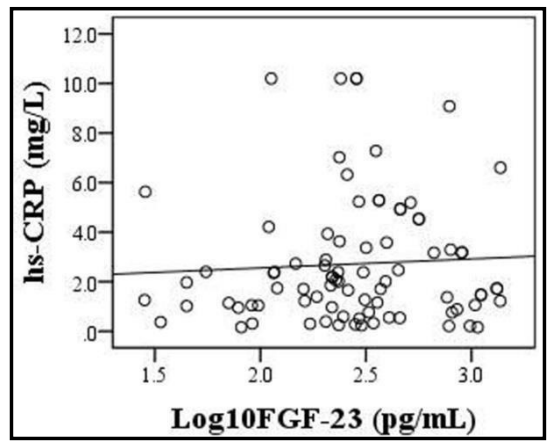

Fig. 7. Correlation between FGF-23 andCasXPs product $(\mathrm{R}=$ $0.006, \mathrm{p}=0.954)$.

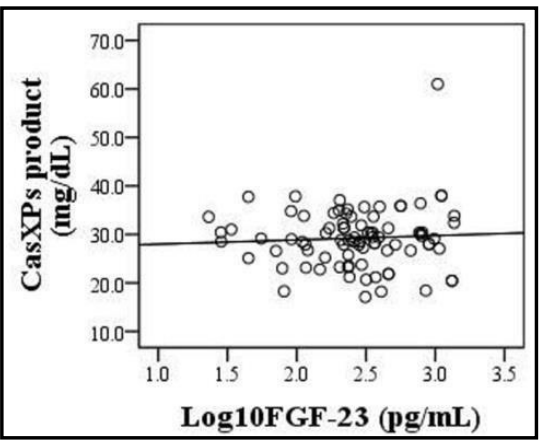




\section{Kidney Blood Pressure Research}

Kidney Blood Press Res 2018;43:1160-1173

\begin{tabular}{l|cl}
\hline DOI: 10.1159/000492244 & ( 2018 The Author(s). Published by S. Karger AG, Basel
\end{tabular} Published online: 31 July, 2018 www.karger.com/kbr

Coban et al.: Fibroblast Growth Factor 23 Levels in Autosomal Dominant Polycystic Kidney Disease

Fig. 8. Correlation between FGF-23 and 25(OH)VitD3 $(\mathrm{R}=0.078$, $\mathrm{p}=0.482$ ).

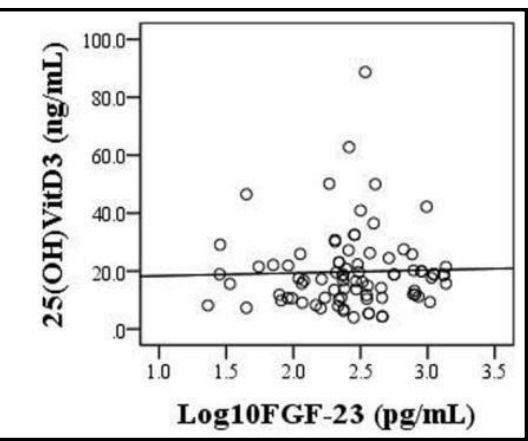

Table 4. Association between serum log10FGF-23 levels and AS and atherosclerosis in patient and healthy control group. Spearman rank test, Pearson correlation test

\begin{tabular}{|c|c|c|c|c|}
\hline \multirow[t]{2}{*}{ Parameter } & \multicolumn{2}{|c|}{$\begin{array}{l}\text { Patients }(\mathrm{n}=86) \\
\text { Log10FGF23 }\end{array}$} & \multicolumn{2}{|c|}{$\begin{array}{l}\text { Healthy control group }(\mathrm{n}=47) \\
\text { Log10FGF23 }\end{array}$} \\
\hline & $\mathrm{r}$ & $\mathrm{p}$ & $\mathrm{r}$ & $\mathrm{p}$ \\
\hline Creatinine $(\mathrm{mg} / \mathrm{dL})$ & 0.433 & $<0.001$ & 0.074 & 0.043 \\
\hline eGFR $\left(\mathrm{mL} / \mathrm{min} / 1.73 \mathrm{~m}^{2}\right)$ & -0.438 & $<0.001$ & -0.333 & 0.022 \\
\hline hs-CRP (mg/L) & 0.076 & 0.489 & 0.279 & 0.058 \\
\hline iPTH (pg/mL) & 0.445 & $<0.001$ & -0.021 & 0.887 \\
\hline CasXPs product (mg/dL) & 0.006 & 0.954 & 0.138 & 0.357 \\
\hline $25(\mathrm{OH}) \mathrm{VitD} 3(\mathrm{ng} / \mathrm{mL})$ & 0.078 & 0.482 & -0.040 & 0.789 \\
\hline $1.25(\mathrm{OH}) 2 \mathrm{VitD} 3(\mathrm{ng} / \mathrm{mL})$ & 0.481 & $<0.001$ & 0.144 & 0.333 \\
\hline baPWV (m/sec) & 0.337 & 0.002 & 0.210 & 0.156 \\
\hline CA-IMT (mm) & 0.298 & 0.005 & 0.019 & 0.899 \\
\hline
\end{tabular}

Fig. 9. Comparison of baPWV values according to mean $s-\mathrm{KL}$ levels ( $\mathrm{p}=0.742)$.

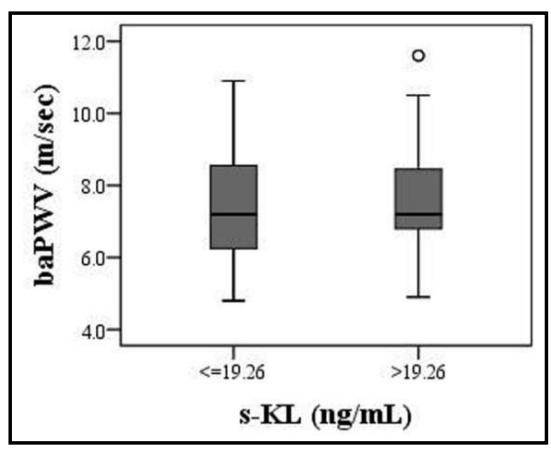

Fig. 10. Comparison of CA-IMT values according to mean s-KL levels ( $p=0.989)$.

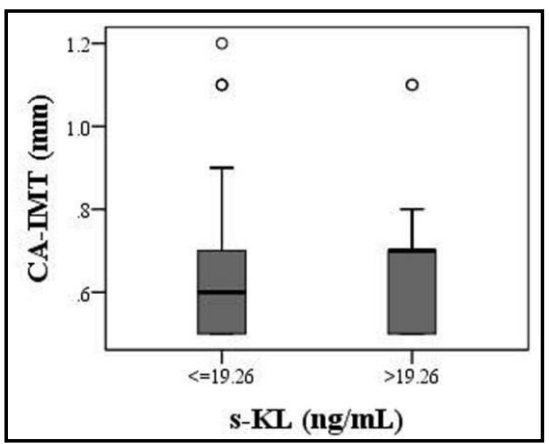




\section{Kidney Blood Pressure Research}

Coban et al.: Fibroblast Growth Factor 23 Levels in Autosomal Dominant Polycystic Kidney Disease

Table 5. Comparison of patients characteristics according to mean s-KLvalues. Data are presented as median (min-max). Mann-Whitney U test

\begin{tabular}{lccc}
\hline Parameter & $\begin{array}{c}\mathrm{s}-\mathrm{KL} \leq 19.26 \mathrm{ng} / \mathrm{mL} \\
(\mathrm{n}=47)\end{array}$ & $\begin{array}{c}\mathrm{s}-\mathrm{KL}>19.26 \mathrm{ng} / \mathrm{mL} \\
(\mathrm{n}=39)\end{array}$ & $\mathrm{p}$-value \\
\hline Creatinine (mg/dL) & $1.32(0.7-4.1)$ & $1.28(0.6-4.19)$ & 0.671 \\
eGFR (mL/min/1.73 m $\left.{ }^{2}\right)$ & $58(17-120)$ & $63(16-122)$ & 0.430 \\
hs-CRP (mg/L) & $2.35(0.16-10.2)$ & $1.72(0.16-10.2)$ & 0.557 \\
iPTH (pg/mL) & $91(18-219)$ & $59(16-185)$ & 0.075 \\
CasXPs product (mg/dL) & $30.08(18.2-37.84)$ & $28.2(17.1-61)$ & 0.480 \\
baPWV (m/sec) & $7.2(4.8-10.9)$ & $7.2(4.9-11.6)$ & 0.742 \\
CA-IMT (mm) & $0.7(0.5-1.1)$ & $0.6(0.5-1.2)$ & 0.989 \\
\hline
\end{tabular}

\section{Discussion}

FGF-23; a phosphatonin that is synthesized from osteocytes and osteoblasts, and plays role in maintaining normal $P$ balance. In advanced stage CKD patients, serum FGF-23 levels are elevated to maintain serum $P$ balance, and this increase is accompanied by increase in serum iPTH levels and decrease in 1.25(OH)2VitD3 levels. s-KL gene is found in bone tissue, as well as in kidneys, parathyroid gland and other endocrine organs. In the presence of its coreceptor s-KL, FGF-23 acts by binding to renal FGF receptors. The aim of our study was to investigate the relationship between serum FGF-23 levels and development of the CV complications AS and atherosclerosis in adult ADPKD patients at various stages of CKD.

In CKD patients, as eGFR value decreases, there is an increase in serum FGF-23 levels and a decrease in serum s-KL levels. Gutierrez et al. reported that an increase in serum FGF-23 levels was observed in patients with CKD to maintain normal serum P balance as eGFR values started to decrease [4]. Fliser et al. [23] reported that increased serum $P$ levels in patients with CKD was accompanied by elevated serum FGF-23 levels. Liu et al. [3] reported that in CKD patients, as the disease stage advanced, the increase in serum FGF-23 and iPTH levels was accompanied by a decrease in serum 1.25(OH)2VitD3 levels. John et al. [5] reported that serum FGF-23 levels were increased in CKD patients in order to decrease the elevated $P$ levels per nephron. Spichtig et al. [24. showed that FGF-23 was synthesized in polcystic mice from the epithelium lining the renal cysts, and that serum FGF-23 levels began to rise from the early stages of renal failure. Mirams et al. [25] reported that FGF-23 was synthesized in ADPKD patients from the cysts present in kidneys and liver. Akiyama et al. [26] suggested that elevated serum FGF-23 levels in ADPKD patients was independent of CKD stage. Pavik et al. [23] showed that serum FGF-23 levels in ADPKD patients were significantly higher compared to studies in CKD patients, and reported lower levels of serum s-KL in ADPKD patients compared to healthy subjects [27]. In our study, a significant increase in serum FGF-23 levels and a significant decrease in serum s-KL levels were observed as the CKD stage advanced in predialysis ADPKD patients. In addition, patients with ADPKD had higher serum FGF-23 and lower s-KL levels than healthy subjects.

PWV measurement from aorta and its main branches is regarded as the gold standard for assessment of AS. The determination of the development of AS by baPWV is considered to be of the same value as other methods. In CKD patients, as CKD stage advances, there is increase in the development of AS. Morimoto et al. [28] reported increased AS development in CKD patients. Heffernan et al. [29] reported increased peripheral vascular dysfunction and increased AS development in early stage ADPKD patients. Yildiz et al. [6] reported increased AS development in early stage ADPKD patients with normal creatinine levels compared to healthy individuals. Borresen et al. [10] reported increased AS development in ADPKD patients with normal creatinine and blood pressure in comparison to healthy subjects. Nowak et al. [30] reported increased AS development starting from the early stages in ADPKD patients. Kocyigit et al. [9] reported increased AS as determined by PWV in ADPKD patients starting from the early stages of the disease. 


\section{Kidney Blood Pressure Research}

Kidney Blood Press Res 2018;43:1160-1173

\begin{tabular}{l|l}
\hline DOI: $10.1159 / 000492244$ & (C) 2018 The Author(s). Published by S. Karger AG, Base
\end{tabular}

Published online: 31 July, 2018

www.karger.com/kb

1170

It is believed that there is no association between increased serum FGF-23 levels and AS development in predialysis CKD patients Ford et al. [13] reported that there was no relationship between increased serum FGF-23 levels AS development as determined by PWV among predialysis CKD patients. Manghat et al. [12] suggested that there was no association between increased serum FGF-23 levels and AS development in predialysis CKD patients, and that the effect of increased FGF-23 in the predialysis period on the CV system was independent of its effects on the vessels. In contrast to all these studies, our study showed that as the CKD stage advanced in ADPKD patients, AS development as determined by baPWV increased when compared to patients at early stage and healthy individuals. Patients with high FGF-23 levels were found to have increased AS development compared to patients with low FGF-23 levels. There was a significant correlation between FGF-23 and AS development, which was independent of s-KL. The difference in the results can be explained by the differences in CKD etiology.

Nowak et al. [30] reported that atherosclerosis development rate in young adult patients with ADPKD was similar to that of healthy young adults. Turkmen et al. [17. reported that atherosclerosis development as determined by CA-IMT was increased in ADPKD patients starting from the early stages Martinez et al. [31] reported increased atherosclerosis development in ADPKD patients with normal creatinine levels. Widlansky et al. [32] reported increased atherosclerosis development in ADPKD patients, which was independent of the increase in blood pressure. Wang et al. [33] reported increased atherosclerosis development as determined by increased CA-IMT in patients with ADPKD who were ad advanced age.

Ashikaga et al. [34] reported that there was no relationship between atherosclerosis development and increased serum FGF-23 levels in CKD patients undergoing dialysis. On the contrary, in their mouse study, Jimbo et al. [35] showed that increased serum FGF-23 levels in the presence of KL led to atherosclerosis development. Nasrallah et al. [36] reported an association between increased serum FGF-23 levels and atherosclerosis development in CKD patients undergoing dialysis. Our study showed that as the CKD stage advanced in ADPKD patients, atherosclerosis development as determined by CA-IMT increased when compared to patients at early stage and healthy individuals. Patients with high FGF-23 levels were found to have increased atherosclerosis development as determined by CA-IMT compared to patients with low FGF-23 levels. There was a significant correlation between increased serum FGF-23 levels and atherosclerosis development in ADPKD patients, which was independent of s-KL.

There are some limitations that have adverse effects on the studyresults. First, the study was conducted in a single center and with low number of patients. Second, since the study was conducted at a cross-sectional time, the long-term effects of increased serum FGF-23 levels on AS and atherosclerosis development in ADPKD patients could not be investigated. Third, patient characteristics were compared to healthy individuals, however, they were not compared to patients with similar CKD stage that developed due to different etiologies. Fourth, since patients continued their medications during the study period, it was not possible to investigate the negative effects of factors such as HT and hyperphosphatemia, which contribute to AS development. Fifth, AS development was assessed with baPWV instrument, however, patients were not evaluated with carotid-femoral PWV instrument, which is known to have higher sensitivity and is regarded as gold standard. Sixth the effect of FGF and its receptors on the vascular cells was not investigated with advanced pathological examination in ADPKD patients.

\section{Conclusion}

As CKD stage in ADPKD patients advanced, serum FGF-23 levels increased and s-KL levels decreased. Advanced stage ADPKD patients were found to have increased AS and atherosclerosis development compared to healthy individuals and patients with early stage 


\section{Kidney \\ Blood Pressure Research}

Kidney Blood Press Res 2018;43:1160-1173

\begin{tabular}{l|l}
\hline DOI: $10.1159 / 000492244$ & (C) 2018 The Author(s). Published by S. Karger AG, Basel
\end{tabular}

Published online: 31 July, 2018

www.karger.com/kbr

disease. In ADPKD patients, increased serum FGF-23 levels showed correlation with AS and atherosclerosis development. In their mouse study, Lindberg et al. [37] showed that arterial s- KL synthesis was very low or not present at all. Donate et al. [38] reported that unlike its effects on kidney and parathyroid gland, the effect of FGF-23 on myocardial cells was independent of s-KL. Our study showed that increased serum FGF-23 levels in ADPKD patients was associated with increased AS and atherosclerosis development, regardless of s-KL. As stated by Faul et al. [39], this suggests that the effect of FGF-23 on peripheral vessels may be independent of s-KL, as is the case with myocardial cells. Nevertheless, there is a need for future larger-scale, multi-centered randomized-controlled clinical and pathological studies investigating the association between increased serum FGF-23 levels and AS and atherosclerosis development in peripheral vessels, and the underlying mechanisms.

\section{Disclosure Statement}

The authors declare that they have no competing interests.

\section{References}

1 Fick-Brosnahan GM, Ecder T, Schrier RW: Polycystic kidney disease; in: Schrier RW (ed): Diseases of the Kidney and Urinary Tract (7th ed). Philadelphia. Lippincott, Williams \& Wilkins, 2001, vol. 20, pp 547-588.

- Kuro-o M, Matsumura Y, Aizawa H, Kawaguchi H, Suga T, Utsugi T, Ohyama Y, Kurabayashi M, Kaname T, Kume E, Iwasaki H, Iida A, Shiraki-Iida T, Nishikawa S, Nagai R, Nabeshima YI: Mutation of the mouse klotho gene leads to a syndrome resembling ageing. Nature 1997;390:45-51.

-3 Liu S, Quarles LD: How fibroblast growth factor 23 works. J Am Soc Nephrol 2007;18:1637-1647.

-4 Gutierrez O, Isakova T, Rhee E, Shah A, Holmes J, Collerone G, Jüppner H, Wolf M: Fibroblast growth factor-23 mitigates hyperphosphatemia but accentuates calcitriol deficiency in chronic kidney disease. J Am Soc Nephrol 2005;16:2205-2215.

5 John GB, Cheng CY, Kuro-o M: Role of Klotho in aging, phosphate metabolism, and CKD. Am J Kidney Dis 2011;58:127-134.

6 Yildiz A, Gul CB, Ersoy A, Asiltas B, Ermurat S, Dogan S, Karaagac K, Sag S, Oruc A, Aktas N, Ocakoglu G, Dogan I, Gullulu S, Gullulu M: Arterial dysfunction in early autosomal dominant polycystic kidney disease independent of fibroblast growth factor 23. Iran J Kidney Dis 2014;8:443-449.

7 Covic A, Florea L, Gusbeth-Tatomir P, Goldsmith DJ: Arterial stiffness in renal patients: An update. Am J Kidney Dis 2005;45:965-977.

-8 London GM, Guerin AP Marchais SJ, Pannier B, Safar ME, Day M, Metivier F: Cardiac and arterial interactions in end-stage renal disease. Kidney Int 1996;50:600-608.

-9 Kocyigit I, Kaya MG, Orscelik O, Kaya C, Akpek M, Zengin H, Sipahioglu MH, Unal A, Yilmaz MI, Tokgoz B, Oymak 0, Axelsson J: Early arterial stiffness and inflammatory bio- markers in normotensive polycystic kidney disease patients. Am J Nephrol 2012;36:11-18.

10 Borresen ML, Wang D, Strandgaard S: Pulse wave reflection is amplified in normotensive patients with autosomal-dominant polycystic kidney disease and normal renal function. Am J Nephrol 2007;27:240-246.

$>11$ Scialla JJ, Wolf M: Roles of phosphate and fibroblast growth factor 23 in cardiovascular disease. Nat Rev Nephrol 2014;10: 268-278.

12 Manghat P, Souleimanova I, Cheung J, Wierzbicki AS, Harrington DJ, Shearer MJ, Chowienczyk P, Fogelman I, Nerlander M, Goldsmith D, Hampson G: Association of bone turnover markers and arterial stiffness in predialysis chronic kidney disease (CKD). Bone 2011;48:1127-1132.

13 Ford ML, Smith ER, Tomlinson LA, Chatterjee PK, Rajkumar C, Holt SG: FGF-23 and osteoprotegerin are independently associated with myocardial damage in chronic kidney disease stages 3 and 4 . Another link between chronic kidney disease-mineral bone disorder and the heart. Nephrol Dial Transplant 2012;27:727-733. 


\section{Kidney \\ Blood Pressure Research}

Kidney Blood Press Res 2018;43:1160-1173

\begin{tabular}{l|l}
\hline DOI: $10.1159 / 000492244$ & (C) 2018 The Author(s). Published by S. Karger AG, Basel
\end{tabular}

Published online: 31 July, 2018 www.karger.com/kbr

14 Poredos P: Intima-media thickness: indicator of cardiovascular risk and measure of the extent of atherosclerosis. Vasc Med 2004;9:46-54.

15 Kocaman O, Oflaz H, Yekeler E, Dursun M, Erdogan D, Demirel S, Alisir S, Turgut F, Mercanoglu F, Ecder T: Endothelial dysfunction and increased carotid intima-media thickness in patients with autosomal dominant polycystic kidney disease. Am J Kidney Dis 2004;43:854-860.

16 Sag S, Yildiz A, Gullulu S, Gungoren F, Ozdemir B, Cegilli E, Oruc A, Ersoy A, Gullulu M: Early atherosclerosis in normotensive patients with autosomal dominant polycystic kidney disease: the relation between epicardial adipose tissue thickness and carotid intima-media thickness. Springerplus 2016;5:211.

-17 Turkmen K, Oflaz H, Uslu B, Cimen AO, Elitok A, Kasikcioglu E, Alisir S, Tufan F, Namli S, Uysal M, Ecder T: Coronary flow velocity reserve and carotid intima media thickness in patients with autosomal dominant polycystic kidney disease: from impaired tubules to impaired carotid and coronary arteries. Clin J Am Soc Nephrol 2008;3:986-991.

18 Larsson T, Marsell R, Schipani E, Ohlsson C, Ljunggren O, Tenenhouse HS, Jüppner H, Jonsson KB: Transgenic mice expressing fibroblast growth factor 23 under the control of the alpha1(I) collagen promoter exhibit growth retardation, osteomalacia, and disturbedphosphate homeostasis. Endocrinology 2004;145:3087-3094.

19 Scialla JJ, Lau WL, Reilly MP, Isakova T, Yang HY, Crouthamel MH, Chavkin NW, Rahman M, Wahl P, Amaral AP, Hamano T, Master SR, Nessel L, Chai B, Xie D, Kallem RR, Chen J, Lash JP, Kusek JW, Budoff MJ, et al.: Chronic Renal Insufficiency Cohort Study Investigators. Fibroblast growth factor 23 is not associated with and does not induce arterial calcification. Kidney Int 2013;83:1159-1168.

-20 Nakayama M, Kaizu Y, Nagata M, Ura Y, Ikeda H, Shimamoto S, Kuma K: Fibroblast growth factor 23 is associated with carotid artery calcification in chronic kidney disease patients not undergoing dialysis: a cross-sectional study. BMC Nephrol 2013;14:22.

-21 Levey AS, Bosch JP, Lewis JB, Greene T, Rogers N, Roth D: A more accurate method to estimate glomerular filtration rate from serum creatinine: a new prediction equation. Modification of Diet in Renal Disease Study Group. Ann Intern Med 1999;130:461-470.

-22 Yokoyama H, Shoji T, Kimoto E, Shinohara K, Kanaka S, Koyama H, Emoto M, Nishizawa Y: Pulse wave velocity in lower-limb arteries among diabetic patients with peripheral arterial disease. J Atheroscler Thromb 2003;10:253-258.

23 Fliser D, Kollerits B, Neyer U, Ankerst DP, Lhotta K, Lingenhel A, Ritz E, Kronenberg F, MMKD Study Group, Kuen E, König P, Kraatz G, Mann JF, Müller GA, Köhler H, Riegler P: Fibroblast growth factor 23 (FGF23) predicts progression of chronic kidney disease: the Mild to Moderate Kidney Disease (MMKD) Study. J Am Soc Nephrol 2007;18:2600-2608.

-24 Spichtig D, Zhang H, Mohebbi N, Pavik I, Petzold K, Stange G, Saleh L, Edenhofer I, Segerer S, Biber J, Jaeger P, Serra AL, Wagner CA: Renal expression of FGF23 and peripheral resistance to elevated FGF23 in rodent models of polycystic kidney disease. Kidney Int 2014;85:1340-1350.

25 Mirams M, Robinson BG, Mason RS, Nelson AE: Bone as a source of FGF23: regulation by phosphate? Bone 2004;35:1192-1199.

-26 Akiyama K, Mochizuki T, Kataoka H, Tsuchiya K, Nitta K: Fibroblast growth factor 23 and soluble Klotho in patients with autosomal dominant polycystic kidney disease. Nephrology (Carlton) 2017;22:848-853.

-27 Pavik I, Jaeger P, Ebner L, Poster D, Krauer F, Kistler AD, Rentsch K, Andreisek G, Wagner CA, Devuyst O, Wüthrich RP, Schmid C, Serra AL: Soluble klotho and autosomal dominant polycystic kidney disease. Clin J Am Soc Nephrol 2012;7:248-257.

-28 Morimoto S, Yurugi T, Aota Y, Sakuma T, Jo F, Nishikawa M, Iwasaka T, Maki K: Prognostic significance of ankle-brachial index, brachial-ankle pulse wave velocity, flow- mediated dilation, and nitroglycerinmediated dilation in end-stage renal disease. Am J Nephrol 2009;30:55-63.

-29 Heffernan KS, Kuvin JT, Sarnak MJ, Perrone RD, Miskulin DC, Rudym D, Chandra P, Karas RH, Menon V: Peripheral augmentation index and vascular inflammation in autosomal dominant polycystic kidney disease. Nephrol Dial Transplant 2011;26:2515-2521.

-30 Nowak KL, Farmer H, Cadnapaphornchai MA, Gitomer B, Chonchol M: Vascular dysfunction in children and young adults with autosomal dominant polycystic kidney disease. Nephrol Dial Transplant 2017;32:342347. 


\section{Kidney \\ Blood Pressure Research}

Kidney Blood Press Res 2018;43:1160-1173

\begin{tabular}{l|l}
\hline DOI: $10.1159 / 000492244$ & (C) 2018 The Author(s). Published by S. Karger AG, Basel
\end{tabular}

Published online: 31 July, 2018

www.karger.com $/ \mathrm{kbr}$

Coban et al.: Fibroblast Growth Factor 23 Levels in Autosomal Dominant Polycystic Kidney Disease

31 Nowak KL, Farmer H, Cadnapaphornchai MA, Gitomer B, Chonchol M: Vascular dysfunction in children and young adults with autosomal dominant polycystic kidney disease. Nephrol Dial Transplant. 2017; 32: 342 347.

-32 Martinez-Vea A, Bardaji A, Gutierrez C, Garcia C, Peralta C, Marcas L, Oliver JA: Exercise blood pressure,cardiac structure, and diastolic function in young normotensive patients with polycystic kidney disease: a prehypertensive state. Am J Kidney Dis 2004;44:216-223.

-33 Widlansky ME, Gokce N, Keaney JF Jr, Vita JA: The clinical implications of endothelial dysfunction. J Am Coll Cardiol 2003; 42:1149-1160.

34 Wang D, Iversen J, Wilcox CS, Strandgaard S: Endothelial dysfunction and reduced nitric oxide in resistance arteries in autosomal-dominant polycystic kidney disease. Kidney Int 2003;64:1381-1388.

35 Ashikaga E, Honda H, Suzuki H, Hosaka N, Hirai Y, Sanada D, Nakamura M, Nagai H, Matsumoto K, Kato N, Mukai M, Watanabe M, Takahashi K, Shishido K, Akizawa T: Impact of Fibroblast Growth Factor 23 on Lipids and Atherosclerosis in Hemodialysis Patients.Therapeutic Apheresis and Dialysis. Ther Apher Dial 2010;14:315-322.

-36 Jimbo R, Kawakami-Mori F, Mu S, Hirohama D, Majtan B, Shimizu Y, Yatomi Y, Fukumoto S, Fujita T, Shimosawa T: Fibroblast growth factor 23 accelerates phosphate- induced vascular calcification in the absence of Klotho deficiency. Kidney Int 2014;85:1103-1111.

-37 Nasrallah MM, El-Shehaby AR, Salem MM, Osman NA, El Sheikh E, Sharaf El Din UA: Fibroblast growth factor-23 (FGF-23) is independently correlated to aortic calcification in haemodialysis patients. Nephrol Dial Transplant 2010;25:2679-2685.

-38 Lindberg K, Olauson H, Amin R, Ponnusamy A, Goetz R, Taylor RF, Mohammadi M, Canfield A, Kublickiene K, Larsson TE: Arterial klotho expression and FGF23 effects on vascular calcification and function. PLoS One 2013;8:e60658.

-39 Donate-Correa J, Mora-Fernandez C, Martinez-Sanz R, Muros-de-Fuentes M. Pérez H, Meneses-Pérez B, Cazaña-Pérez V, Navarro-González J: Expression of FGF23/KLOTHO system in human vascular tissue. Int J Cardiol 2013;165:179-183.

-40 Faul C, Amaral AP, Oskouei B, Hu MC, Sloan A, Isakova T, Gutiérrez OM, Aguillon-Prada R, Lincoln J, Hare JM, Mundel P, Morales A, Scialla J, Fischer M, Soliman EZ, Chen J, Go AS, Rosas SE, Nessel L, Townsend RR, et al.: FGF23 induces left ventricular hypertrophy. J Clin Invest 2011;121:4393-4408. 